\title{
¿CUÁNTOS CÁNCERES PRECOCES DE PRÓSTATA ES NECESARIO DETECTAR PARA PREVENIR UN FALLECIMIENTO?
}

\author{
Jorge Valle Gerhold.
}

Servicio de Urología. Hospital Clínico Universitario Zaragoza. Zaragoza. España.

\begin{abstract}
Resumen.- OBJETIVO: Conocer la significancia clínica del diagnóstico y tratamiento del cáncer de próstata diagnosticado en pacientes asintomáticos, en términos de supervivencia y enfrentarla a los inconvenientes.

MÉTODOS: Se revisan en la bibliografía los datos actuales sobre la cantidad de varones que es preciso revisar mediante análisis del PSA, biopsiar y operar para prolongar la vida a uno de ellos, así como los inconvenientes derivados del proceso.
\end{abstract}

RESULTADOS: Se resume que en el caso de los varones mayores de 50 años, 1 de cada 9 tendrá el PSA mayor a $4 \mathrm{ngr} / \mathrm{ml}$. Será necesario biopsiar a 3 varones si el PSA está entre 4 y $10 \mathrm{ngr} / \mathrm{ml}$ para encontrar un ade-

\section{CORRESPONDENCIA}

Jorge Valle Gerhold

Hospital Clínico Universitario Zaragoza

Av. San Juan Bosco, 15

50009 Zaragoza (España)

vallegerhold@gmail.com

Aceptado para publicar: 12 de marzo 2010 nocarcinoma de próstata. Se prolongará la vida a un paciente de cada 18,5 prostatectomías si el tumor es palpable y se estima un número necesario cinco veces mayor para los no palpables. Como contrapartida, se producirá una muerte por cada 9791 varones revisados, una incontinencia por cada 147 varones revisados y una impotencia por cada 58 varones revisados. Se exponen 3 publicaciones sobre expansión tumoral provocada por las biopsias.

CONCLUSIONES: Se destaca la escasa evidencia de los datos en términos de supervivencia, a pesar de los años transcurridos desde que se difundió el uso del PSA. Se advierte sobre la diferencia de resultados entre los centros de excelencia y el resto y sobre las dudas que crea la definición de cáncer cuando éste no puede objetivarse más allá de la biopsia.

Ante estas cifras, se pone en duda la utilidad de la búsqueda y tratamiento de cánceres precoces de la próstata en términos de coste-beneficio, recomendándose la transmisión de esta información a los pacientes, para que la tengan en cuenta antes de decidirse por una u otra opción. Destaca la necesidad de investigaciones prospectivas a largo plazo para aclarar los casos en los que se hace necesario intervenir y evitar el sobrediagnóstico y se invita a reflexionar sobre la idoneidad de la búsqueda a ultranza de hallazgos patológicos en varones cada vez más jóvenes y con límites del PSA más bajos.

Palabras clave: Cáncer de próstata. Sobrediagnóstico. Prostatectomía. Supervivencia. Cáncer precoz.

Summary.- OBJECTIVES: To determine the clinical significance of diagnosis and treatment of prostate cancer diagnosed in asymptomatic patients, in terms of survival and to face its disadvantages. 
METHODS: We reviewed the literature in order to know the number of men that must be reviewed through analysis of PSA, biopsy and surgery to prevent the death of one of them, as well as knowing the amount of disadvantages associated with the process.

RESULTS: We summarized, in the case of men older than 50 years, that 1 in 9 have PSA greater than 4 $\mathrm{ng} / \mathrm{ml}$. We must biopsy 3 men if PSA is between $4-10$ $\mathrm{ng} / \mathrm{ml}$ to find one prostate cancer. In order to prolong the life of one patient it is necessary a total of 18.5 prostatectomies if the tumor is palpable and the number of prostatectomies needed in the case of non-palpable tumors is estimated five times higher. In return, there will be one death per 9791 males initially checked, one incontinence per 147 males and one impotence per 58 male impotence. Three papers report that tumor spread may be caused by the biopsies.

CONCLUSIONS: We highlight the limited evidence for the data in terms of survival. It warns about the difference in results between the centers of excellence and the rest, and creates doubts about the definition of cancer when it cannot be demonstrated beyond the biopsy.

The usefulness of finding and treating early prostate cancers is questionable in terms of cost-benefit, recommending the transfer of this information to patients, before deciding on one or another option. We stress the need for long-term prospective investigations to clarify which cases need to be treated and to prevent overdiagnosis. We should consider whether it is worth looking further histological findings, in increasingly younger males and with lower PSA limits.

Keywords: Prostate cancer. Overdiagnosis. Prostatectomy. Survival. Early cancer.

\section{INTRODUCCIÓN}

Hace más de 20 años, la mayor parte de los cánceres de próstata se descubrían cuando su extensión local o a distancia hacían imposible cualquier tratamiento que pretendiera erradicarlo. A partir de los años 90, con el advenimiento de los análisis sistemáticos del PSA y el tacto rectal (TR), se dispararon los diagnósticos de cánceres en fase precoz. En su mayor parte se trataba de hallazgos en los cilindros de biopsia de tamaño insuficiente como para producir un nódulo palpable o provocar cualquier tipo de síntoma. Se añadió a la clasificación TNM el Tlc, dando por sentado que el haberlo detectado mediante elevación del PSA ya implicaba una historia natural diferente. Una vez detectados, se podían eliminar mediante cirugía o radioterapia, esperando que de este modo, la aparición de metástasis, y por tanto la mortalidad inherente al cáncer de próstata, disminuyera drásticamente en las décadas siguientes sobre las poblaciones estudiadas.

Sin embargo, las publicaciones donde se concluya una mejoría incuestionable en la supervivencia no acaban de llegar. En su lugar, todavía se multiplican las que hacen referencia al modo de diagnosticar el número mayor posible de nuevos cánceres o las que evalúan los resultados de los distintos tratamientos en base a parámetros indirectos, como el PSA. Mientras para muchos grupos sigue siendo razonable rebajar la edad de los pacientes y las cifras de PSA que aconsejen la biopsia, a otros clínicos se nos hace necesario resumir los datos de que disponemos en relación a la supervivencia y asumir las incógnitas pendientes, para así poder transmitir ambos aspectos a nuestros pacientes, que han de ser en definitiva los que tengan la última palabra.

Pero difícilmente vamos a transmitir una información útil a nuestros pacientes y plasmarla en respuestas sencillas si nosotros mismos tenemos dificultades para resumir las ingentes cantidades de información que se publica. En este sentido, no basta con diferencias estadísticamente significativas entre dos variables, se hace preciso aclarar hasta qué punto poseemos evidencias palpables que aconsejen acciones arriesgadas con el mínimo margen de duda. De una forma harto resumida y esquemática, considerando los casos más favorables de cara al intervencionismo, será necesario conocer cuántos varones es preciso estudiar durante un mínimo de años para encontrar un PSA que justifique realizar una biopsia; cuántos de entre aquellos a quienes se realice la biopsia, realmente tendrán un cáncer, y de éstos últimos, cuántos alargarán su vida gracias a nuestros esfuerzos (y su sufrimiento). Además habrá que conocer también (y sopesar) los efectos secundarios y los costes económicos que acarrearán las diferentes maniobras del proceso. Seguidamente vamos a intentar responder a estas preguntas de la forma más simple posible con los datos que pueden localizarse en la bibliografía, bajo los tres epígrafes básicos: el cribado mediante PSA, el valor de la biopsia y las alternativas de tratamiento.

\section{EL PSA EN EL CONTEXTO DE HOMBRES ASIN- TOMÁTICOS}

Las cifras del PSA varían en virtud de una serie de factores, pues se trata de una molécula específica del órgano, no de sus enfermedades. No se conoce una cifra límite definitoria de cáncer o de su ausencia. De todas formas, ésta siempre se ha considerado alrededor de $4 \mathrm{ngr} / \mathrm{ml}$. Con esta cifra como 
referencia, a partir de los 50 años, aproximadamente el $11 \%$ de los varones lo tienen más elevado en la primera visita $(1,2)$. Si se considera en lugar de un único análisis, la tasa de positivos a lo largo de varios años (2 a 4 años), y el punto de corte del PSA considerado positivo se desciende a $3 \mathrm{ngr} / \mathrm{ml}$, al final una media del $16 \%$ lo rebasan (3). Dicho de otro modo, es necesario analizar una vez la sangre de unos 9'09 varones mayores de 50 años para encontrar en uno de ellos el PSA por encima de $4 \mathrm{ngr} / \mathrm{ml}$., - bien puede seguir acudiendo periódicamente para conocer la evolución de las cifras del PSA a lo largo del tiempo, se encontrará elevado por encima de 3 $\mathrm{ngr} / \mathrm{ml}$ en 1 de cada 6 casos.

Dentro de los varones cuyo PSA ha permanecido por debajo, surge la siguiente pregunta: ¿̇cada cuánto tiempo está justificado seguir determinando el PSA? De hecho no existe un periodo definido como el más adecuado que no esté sujeto a polémica. Si el objetivo es diagnosticar cánceres de próstata a ultranza, la respuesta es simple, lo más frecuentemente posible, recomendando la biopsia incluso con cifras de PSA menores a los $4 \mathrm{ngr} / \mathrm{ml}$ estándar. Si por el contrario se consideran criterios de eficiencia y la lenta evolución natural, pueden demorarse mucho más. La diferencia de una u otra actitud es enorme, dado el elevado número de varones a estudiar, ¿̇Cuáles serán los criterios que nos aconsejen un mayor o menor interés en la búsqueda de los cánceres asintomáticos? En este caso, como en la detección precoz de cualquier enfermedad, es necesario que se cumplan una serie de premisas para que sea aconsejable: diagnóstico posible antes de que se manifiesten sus síntomas (sin falsos positivos), dicho diagnóstico debe ser poco traumático y asequible, debe existir un tratamiento eficaz para la enfermedad y dicho tratamiento deberá ser significativamente más eficaz si el diagnóstico es precoz que si se hace cuando el paciente consulta por sí mismo. Los dos artículos aparecidos este año del grupo norteamericano "Prostate, Lung, Colorectal, and Ovarian Cancer Screening Trial project team" (PLCO) y del "European Randomized Study of Screening for Prostate Cancer" (ERSPC) $(3,4)$, han intentado resumir los resultados del cribado en el punto clave, la mortalidad. Esto es muy importante, porque los puntos intermedios como el diagnóstico total de cánceres o la fase clínica de los mismos pueden ayudar a orientar los siguientes pasos a los grupos investigadores, pero lo que de verdad sirve para decidir si vale o no la pena, es conocer si sometiéndose al TR y al análisis del PSA vivirán más tiempo. En este sentido, el PLCO no ha conseguido demostrar diferencias significativas entre los grupos de cribado y de control, aunque tampoco han quedado descartadas, los mismos autores explican que algunos problemas pudieron enmascarar una ligera ventaja en la supervivencia del grupo sometido a cribado, especialmente por el hecho de que casi la mitad del grupo control decidieron, a pesar de todo, someterse a TR y analizar su PSA y uno de cada seis del grupo de cribado lo abandonaron antes de concluir. Por otra parte, la inclusión de la mortalidad inherente a los tratamientos, dentro de la causada por el propio tumor o la siempre discutible certificación de las causas de la muerte podrían haber sesgado los resultados a favor del grupo de cribado (5). En cambio, el ERSPC sí encontró diferencias significativas, al menos estadísticamente, dando ventaja al programa de detección precoz para prevenir alguna de las muertes debidas al cáncer de próstata. Pero el problema se centra en la significancia clínica, y con un riesgo absoluto de $0^{\prime} 71$, se necesitan incluir a 1410 varones para poder decir que han prevenido la muerte de uno de ellos, incluyendo entre otros muchos costes, la necesidad de aplicar algún tratamiento a 48 varones por cada varón beneficiado (Tabla I).

A esto se añade que en las dos series existen algunas dificultades que pueden afectar notoriamente los resultados, especialmente en lo referente a los tratamientos aplicados, donde no consta que se distribuyeran homogéneamente entre los grupos a comparar. De hecho, en el ERSPC se constatan diferencias notables cuando, una vez diagnosticados del cáncer y en la misma situación del TNM, en el grupo de cribado se han practicado muchas más cirugías y el grupo control mucha más hormonoterapia (véase el apéndice de la misma publicación). En términos absolutos podría aducirse que los diagnosticados en el grupo estudio llegan en una fase más precoz, y por tanto hay más oportunidades para los tratamientos radicales, pero si se analizan las tablas del mencionado apéndice esto no es así, explicándose los resultados tan exiguos. Una publicación posterior del mismo grupo europeo (6) intenta solventar el problema de la elevada tasa de abandonos en el grupo de cribado y de contaminación por PSA en el grupo control. Separan mediante una técnica estadística los varones "cumplidores" con lo que se esperaba de ellos en el estudio (aquellos que se revisaron en el grupo de cribado y los que no se analizaron el PSA en el grupo de control), del resto que no cumplieron (desapareciendo del grupo de cribado y analizándose por su cuenta el PSA si estaban en el grupo de control). De esta forma encuentran una mejoría en el riesgo relativo, pero ésta es difícilmente justificable desde el punto de vista que nos interesa a los clínicos: la decisión de cada varón entre cumplir o no cumplir el programa previsto por los investigadores no se debió al azar y por lo tanto resta homogeneidad a ambos grupos del mismo modo que la restaron las diferencias tan acusadas en la distribución de los tratamientos. 
TABLA I. RESUMEN ESQUEMÁTICO DE LOS DATOS ORIENTATIVOS SOBRE VENTAJAS E INCONVENIENTES A LARGO PLAZO DEL SOMETIMIENTO AL CRIBADO CON PSA A PARTIR DE LOS 50 AÑOS. EXPLICACIÓN Y BIBLIOGRAFÍA EN EL TEXTO.

\begin{tabular}{|l|r|}
\hline \multicolumn{1}{|c|}{ VENTAJAS ESPERABLES DEL CRIBADO } & \\
\hline № varones necesarios para prevenir 1 muerte & 1410 \\
№ varones necesarios para 1 PSA>4ngr/ml a la primera & 9 \\
\hline № varones necesarios para 1 PSA>3ngr/ml durante 4 años de seguimiento & 6 \\
№ biopsias necesarias para encontrar un cáncer & 3 \\
\hline № prostatectomías necesarias para prevenir 1 muerte (nº estimado si T1c) & $18^{\prime} 5$ (>100) \\
\hline INCONVENIENTES & 9791 \\
№ varones revisados para provocar 1 muerte & 58 \\
\hline № varones revisados para provocar 1 con incontinencia & 147 \\
\hline 1410 varones a estudio $\rightarrow 48$ prostatectomías $\rightarrow 1$ muerte prevenida $\leftarrow 24$ impotentes, \\
9 incontinentes, etc
\end{tabular}

\section{LA UTILIDAD DE LA BIOPSIA DE PRÓSTATA}

En el caso de aquellos varones en los que se ha encontrado una elevación anormal del PSA, una vez informados de que el único diagnóstico de certeza del cáncer de próstata lo ofrece la biopsia y antes de decidirse a realizarla, puesto que se trata de una prueba cruenta será recomendable informarles sobre qué probabilidades existen de encontrar un cáncer, así como de las complicaciones que pueden sufrir. Al igual que con el PSA, las probabilidades varían en virtud de una serie de factores como la edad o la técnica de la biopsia entre otros, pero las cifras más importantes pueden resumirse de la siguiente manera (7):

Si no existen hallazgos patológicos en el TR, cuando el PSA se encuentra por encima de $4 \mathrm{ngr} / \mathrm{ml}$, 1 de cada 3 biopsias tendrá cáncer; en el segmento de varones con el PSA >10 ngr/ml (que solo representan el $7 \%$ de los varones analizados), las probabilidades de encontrar un tumor suben hasta el $65 \%$.

La existencia de hallazgos en el TR modifica sustancialmente los resultados, en este caso, cuando el PSA está entre 4 y 10 , las posibilidades son de 1 cáncer de cada 2 biopsias y si el PSA supera los 10 $\mathrm{ngr} / \mathrm{ml}$, alcanzan el $90 \%$.

Las biopsias no presentan a menudo complicaciones importantes: 1 paciente de cada 200 ingre- sará por infección urinaria o sepsis, 1 de cada 200 presentará retención urinaria, 1 de cada 5 tendrá hematuria y 1 de cada 2, hematospermia (8). Además de esto hay que considerar otros inconvenientes difíciles de cuantificar, pero no menos importantes en virtud del elevado número de procedimientos, que tan solo se trata de una prueba diagnóstica y que a menudo se realiza en pacientes asintomáticos: la carga emocional de sufrir una intervención quirúrgica y esperar un resultado temido, el dolor inherente al procedimiento si se realiza sin anestesia, el sometimiento a la anestesia cuando ésta se realiza, el porcentaje variable de ingresos hospitalarios, o el coste económico de todo el proceso.

Ante un informe que concluye con el diagnóstico de adenocarcinoma de próstata, es común informar al paciente afecto que padece un cáncer. Hasta este momento todo parece muy simple, pero el problema llega si dicho paciente nos pregunta si eso significa que va a morir en breve tiempo como consecuencia del mismo, porque evidentemente no suele ser así, incluso si no se aplica ningún tipo de tratamiento. Hay que recordar que existen varios matices que diferencian a éste tumor de otros. En primer lugar, se da con frecuencia en personas ancianas, en las que es normal que surja cualquier otro problema de salud que acabe con su vida antes que el propio tumor. Pero también se añade el hecho de que en pacientes más jóvenes pueden pasar muchos años sin que aparezcan signos de progresión, lo que añade 
otra característica a este tumor: la evolución extraordinariamente lenta con la que evoluciona en un buen número de pacientes. Pero ¿̇cómo podemos anticipar si nos encontramos ante un cáncer de evolución lenta y no ante un hallazgo histológico irrelevante, sin perjuicio de que con los años acabe apareciendo por allí un cáncer clínicamente manifiesto? El mismo paciente puede preguntarse qué clase de cáncer es ése que ni se toca, ni se ve, ni le da problemas, ni probablemente se los vaya a dar en muchos años, incluso sin tratamiento. Queda al descubierto el problema clave de este tumor: del sobrediagnóstico. Si recurrimos a la definición general de cáncer, ésta comporta la existencia de un abultamiento sólido, celular, con capacidad de invasión de tejidos vecinos y de producir metástasis a distancia. El estudio microscópico nos describe el patrón celular que suele encontrarse en los cánceres, pero si no puede demostrarse macroscópica ni clínicamente, parece razonable cuestionarse si realmente se trata de tal. Antes de la época del PSA, ya se catalogaron los hallazgos microscópicos en las próstatas operadas por hiperplasia como $\mathrm{Tla}$ y $\mathrm{T} 1 \mathrm{~b}$, conviniendo que no merecía la pena su tratamiento ulterior más allá de un prudente seguimiento. Incluso hoy en día, en numerosas guías clínicas se sigue recomendando tal postura. $\dot{i}$ En qué se basa pues el reconocimiento como cáncer susceptible de tratamiento el Tlc? Ya antes de la era de PSA, un grupo estudió la relación en la misma población de los diagnósticos en vida de cáncer de próstata y sus consecuencias clínicas, con los hallazgos en próstatas de autopsias realizadas a varones mayores de 50 años, no diagnosticados en vida de cáncer. Al encontrar que 1 de cada 3 autopsias tiene cáncer, llegando a 2 de cada 3 en los mayores de 80 años y que solo 1 de cada 50 fallecidos en la cohorte a estudio fallecieron por el cáncer de próstata, los mismos autores advirtieron sobre los peligros del sobrediagnóstico (9). Realmente es difícil trasladar estas dudas a los pacientes, pero sin duda estamos en el deber de intentarlo: son hallazgos microscópicos coincidentes con los de un cáncer de próstata, pero lo cierto es que no podemos afirmar con rotundidad que éste exista mientras no lo veamos, es decir mientras no se manifieste clínicamente o se pueda palpar o demostrar en alguna prueba radiológica.

Ahondando más en el tema, es muy sugerente el estudio publicado por Chen (10), donde relacionan en modelo experimental con animal que la existencia de determinados genes frena de forma definitiva el crecimiento inicial del cáncer de próstata inducido, convirtiéndolo en un ente histológico latente, del mismo modo que ciertas manchas cutáneas, o lunares, nos acompañan toda la vida sin que nadie se atreva a diagnosticarlos de melanoma. Es muy sugerente que dicho gen "frenador" se encuentre en los tumores prostáticos humanos extirpados de forma "precoz". Según sus hallazgos, las lesiones histológicas portadoras de este gen no serían propiamente un tumor, ni tan siquiera una lesión preneoplásica, no más que un inofensivo lunar.

Otro aspecto poco investigado y que rara vez se menciona, se suma al resto de los efectos secundarios ligados a las biopsias. Se trata de la posibilidad de extensión tumoral. Por motivos anatómicos y técnicos es realmente difícil de cuantificar, pero ya se han publicado casos que demuestran que ésta existe, por lo menos a nivel local en el trayecto de las agujas (1 1-13). Es más difícil demostrar la extensión a distancia, pero no deja de tener su riesgo, especialmente en aquellos tumores más agresivos que son precisamente los que necesitamos curar. No por ser de difícil demostración deja de ser razonable su existencia y debería tenerse en cuenta cuando muchos investigadores optan por extraer un número cada vez mayor de cilindros de las glándulas a estudio y nos hemos permitido olvidar la punción con aguja fina, que también tuvo la oportunidad de demostrar su eficacia (14).

\section{LA DECISIÓN FINAL, EL TRATAMIENTO}

Aquí surge la siguiente pregunta clave, qué probabilidades tendrá el paciente de curarse de su tumor, sufriendo qué tipo de efectos secundarios y cuáles serían las consecuencias en el caso de que decidiera no hacerse nada. La respuesta precisa una serie de pacientes numerosa, distribuidos aleatoriamente en grupos homogéneos para prostatectomía, radioterapia o simple observación, seguidos minuciosamente durante más de 10 años y asegurándose de la causa de muerte con la máxima fiabilidad, incluyendo un porcentaje suficiente de autopsias. A pesar de los miles de pacientes tratados en todo el mundo y las dudas creadas sobre la efectividad de los tratamientos, dicha investigación no se ha publicado todavía en dichos términos. Bajando el nivel de exigencia, podrían ayudar las publicaciones en las que al menos se compare de forma prospectiva la aplicación de un tratamiento con la ausencia del mismo, a largo plazo y en términos de mortalidad causa-específica. Los resultados acerca de marcadores intermedios, como el PSA pudieron ser muy útiles al principio, cuando se comenzó a tratar a estos pacientes veinte años atrás, pero en este momento, tras muchos miles de pacientes operados se hace necesario conocer datos más sólidos: es necesario comprobar si realmente estamos alargando o no la supervivencia. La única serie conocida que cumple en cierta medida estos criterios, comparando la prostatectomía frente a la abstención terapéutica hasta que se hacen nece- 
sarios los cuidados sintomáticos, se ha llevado investigando durante los últimos 12 años en Suecia (15). Se han publicado a lo largo de los últimos años los resultados en términos de supervivencia de los dos grupos de pacientes distribuidos al azar: al primero, de 280 varones tratados mediante prostatectomía y al segundo, de 283, dejado a su evolución espontánea. La principal dificultad para inferir de sus resultados los esperados en los pacientes diagnosticados en el contexto de una campaña de cribado estriba en que el $83,3 \%$ de la serie inicial se trataba de tumores T2, frente al 11,7\% que fueron T1c. Pasados los años, en las curvas de supervivencia ha existido una diferencia a favor de los operados, temporalmente significativa a los 9 años y que finalmente dejó de serlo pasados los 10 años. En cifras absolutas, tras 12 años de seguimiento, el riesgo absoluto de reducir la mortalidad mediante la prostatectomía frente a la observación fue de $5^{\prime} 4 \%$, lo que significa que el número necesario para tratar (NNT) es de 18,5. Es decir, son necesarias 18,5 prostatectomías para prevenir una muerte dentro de los siguientes 12 años de la intervención.

Por otra parte, la cirugía radical de próstata no está exenta de riesgos (16). En las series publicadas con un gran número de casos, existen 3 fallecimientos de cada 1000 intervenciones, la hemorragia media suele superar un litro, y 1 de cada 30 pacientes sufrieron complicaciones cardiopulmonares graves. A largo plazo la incontinencia publicada por centros de excelencia oscila entre el 5 y el $20 \%$, que asciende a tasas entre el 19 y el $31 \%$ en las encuestas realizadas telefónicamente a los pacientes operados en general (17). La impotencia, cuya valoración es muy diferente según las diferentes publicaciones y los antecedentes de los pacientes, en el mejor de los casos afecta a 1 de cada 4 operados, pero suele afectar a bastante más de la mitad.

Evidentemente también existen otros métodos de tratamiento mediante los diferentes usos de las radiaciones ionizantes, pero no se han publicado resultados a largo plazo que enfrenten la radioterapia con la abstención terapéutica y es difícil suponer que puedan mejorar mucho el panorama. Su principal papel hablando de resultados, estriba en disminuir la tasa de impotencia (papel que se relativiza notablemente cuando se aplica castración química de modo adyuvante) y su equiparación en resultados bioquímicos a la cirugía. A pesar de que pueda valorarse de forma optimista al haberse logrado evitando el paso por el quirófano, no deja de confirmar unos resultados tan pobres como los de la propia intervención.

Ante varones operados en base a la elevación de un PSA estando asintomáticos, sería desea- ble demostrar una efectividad amplia de la cirugía para compensar los costes y para ello no es válido que los resultados vuelvan a ser evaluados según suba o baje el PSA, técnicamente se trata de una tautología, careciendo por tanto de rigor científico: si el PSA no es sinónimo de enfermedad, tampoco lo puede ser de curación. La única forma de romper el círculo vicioso y establecer una lógica racional de causas y efectos obliga a comparar datos de supervivencia en grupos operados y sin operar, y una vez demostrada dicha diferencia como estadísticamente significativa, cuantificar los datos para demostrar una superioridad marcadamente favorable al grupo de los prostatectomizados que compense los costes. Sólo de esta forma quedaría establecida una significancia clínica que justificara las ventajas que se atribuyen a las prostatectomías. Pero la serie escandinava apenas encuentra diferencia frente a la abstención terapéutica y eso que tan solo el $11 \% \%$ de los pacientes correspondían a una fase Tlc, puesto que la mayoría eran palpables, es decir, como mínimo T2. Los mismos autores advierten que en el caso de los Tlc, de acuerdo con los datos aportados por su grupo y usando los parámetros clínicos explicados por Klotz (18), el NNT necesario para los Tlc puede multiplicarse por cinco, es decir, podrían ser necesarias más de 100 prostatectomías para prevenir una muerte por cáncer de próstata más allá de los 12 años de seguimiento. En este punto, Klotz aboga por intentar encontrar un camino intermedio, afinando la definición de cánceres de baja agresividad, para limitar en éstos los tratamientos radicales, limitándose a vigilarlos en una suerte de "prueba del tiempo" y evitar así el "infradiagnóstico" de los que padezcan tumores realmente agresivos. Esta postura ecléctica puede tener cierto valor "diplomático" ante la confusión creada tras los abusos del diagnóstico, pero no deja de presentar sus riesgos, y es difícil de sostener sobre datos racionales cuando llega a recomendar biopsias cada cierto tiempo que serían inútiles una vez más para los pacientes sanos y peligrosas para los que no lo son.

En los casos del cáncer de mama o cérvix, ante unas cifras teóricamente similares a las que podemos conocer sobre el cáncer de próstata, los niveles de recomendación para el cribado que otorga la U.S. Preventive Services Task Force son de nivel B para mama, de nivel A para cérvix y ausencia de evidencia para próstata (19-21). Esto tiene mucho que ver con la escasa evidencia de los datos, porque es necesario añadir que los aquí expuestos están en cierto modo sesgados hacia arriba, es decir se han supuesto las situaciones más favorables para que, diagnosticando y tratando precozmente a los pacientes en las circunstancias óptimas, éstos puedan verse beneficiados al máximo, pero en la realidad surgen 
otros problemas que tienden a empeorarlo todo: Buen número de los pacientes, cuando se diagnostican, ya tienen el cáncer en fase avanzada y poco se puede hacer por ellos, añadido a lo que habitualmente se hace cuando acuden a la consulta por causa de los síntomas. Otro grupo de ellos enferma gravemente - fallece en el proceso de diagnóstico por cualquier otro motivo. A otros se les retrasa el diagnóstico o tratamiento de diferentes enfermedades que de verdad les molestan al ser "sospechosos" de un posible cáncer de próstata. Tampoco hay que olvidar que la prostatectomía no deja de ser una intervención que exige una técnica muy refinada para evitar secuelas importantes, es necesario pasar por unas curvas de aprendizaje que difícilmente superan los urólogos de muchos hospitales, por este motivo, los resultados de las encuestas independientes distan mucho de los publicados por los centros de excelencia (17). Todo esto desalienta a médicos y pacientes, buscando otras alternativas como la radioterapia en sus diferentes variantes cuando todavía están pendientes de demostrar su superioridad frente a la abstención terapéutica y ya empiezan a publicarse sus consecuencias adversas a largo plazo (22). Por último, no hay que olvidar que el conjunto de diagnóstico precoz y tratamiento a los varones asintomáticos también tiene un coste-oportunidad dentro de un sistema sanitario de medios limitados, incluso a nivel de los propios afectados, cuando realmente son alcanzados por un cáncer avanzado, pierden oportunidades inmersos en las listas de espera al verse obligado el sistema a atender a los supuestamente enfermos.

\section{CONCLUSIONES}

\section{Para los varones que querrían revisarse su prós- tata:}

Se hace necesario plantear las opciones con claridad, a la vez que con prudencia, considerando las circunstancias de edad, de salud general, de factores bioquímicos o histológicos concomitantes, y reconociendo las limitaciones que actualmente se tienen en nuestros conocimientos. Es muy importante transmitir esta misma prudencia a los medios de comunicación, a la atención primaria y a los médicos del trabajo.

\section{Para los diagnosticados de un cáncer precoz de próstata:}

$\mathrm{Ni}$ vale decirle a secas que tienen un cáncer y debe extirparse lo antes posible, ni tampoco es posible, después de tantos esfuerzos diagnósticos, etiquetarles de sanos y devolverles a su casa. Si se deciden por la cirugía deben conocer los efectos adversos que se esperan en el medio que va a operarles y el NNT. Si se deciden por la radioterapia deben ser informados acerca de la ausencia de datos a largo plazo sobre supervivencia frente a la observación y la posibilidad de nuevos canceres en vejiga o colon inducidos por las radiaciones. Siempre se debe plantear como alternativa real y razonable la simple observación.

\section{Para el sistema sanitario:}

En el comienzo del proceso, cuando se les pide el primer PSA o cuando se opina en los medios de comunicación, es sin duda el momento donde más importancia tiene una correcta información. La abstención es mucho más difícil de aceptar cuando ya se ha sufrido una serie de análisis, exploraciones y biopsias que evidentemente la contradicen.

\section{Para los investigadores:}

Los clínicos necesitamos conocer qué cánceres son tales, necesitamos estudios prospectivos a largo plazo comparando tratamientos frente a ausencia de los mismos, necesitamos métodos para clasificar los cánceres agresivos y disminuir el sobrediagnóstico. No necesitamos más hallazgos histológicos, sino certezas sobre qué tumores son los realmente letales y cual es el tratamiento más adecuado para erradicarlos. Es de esperar que estudios venideros puestos en marcha en este momento nos aporten una luz realmente necesaria para el manejo futuro de esta patología (23).

\section{BIBLIOGRAFÍA y LECTURAS RECOMENDADAS ( ${ }^{*}$ lectura de interés $y^{* *}$ lectura fundamental)}

1. Brawer M K, Chetner MP, Beatie J, and Lange $\mathrm{PH}$. Prostate specific antigen and early detection of prostatic carcinoma. J Urol, 1991; 145: 382-6. Hudson MA, Migliore P, Weinberg A, Hedrik T,

2. Wargo M, and Scardino PT. Use of digital rectal exam, transcendental ultrasonografy and serum PSA in prostate cancer screening. J Urol, 1991; 145: 381-6.

**3. Schröder FH, Hugosson J, Roobol MJ, Tammela TL, Ciatto S, Nelen V, et al. Screening and Prostate-Cancer Mortality in a Randomized European Study; N Engl J Med, 2009; 360:1320-8.

**4. Andriole GL, Crawford ED, Grubb III RL, Buys SS, Chia D, Church TR, et al. Mortality Results from a Randomized Prostate-Cancer Screening Trial; N Engl J Med, 2009; 360:1310-9.

5. Johansson JE, Holmberg L, Johansson S, Bergstrom R, Adami HO. Fifteen-year survival in prostate cancer: a prospective, population-based study in Sweden. JAMA. 1997; 277; 467-71.

6. Roobol MJ, Kerkhof M, Fritz H, Schröder FH. Prostate Cancer Mortality Reduction by Prostate- 
Specific Antigen-Based Screening Adjusted for Nonattendance and Contamination in the European Randomised Study of Screening for Prostate Cancer (ERSPC) Eur Urol, 2009; 56: 584591.

7. Ballentine Carter H, Partin AW. En Campbell's Urology, ed. W.B. Saunders Company, Philadelphia, 1998, $7^{\mathrm{a}}$ ed. pag 2521- 2522

8. Raaijmakers R, Roobol MJ, Wildhagen MF, Complication rates and risk factors of 5802 transrectal ultrasound-guided sextant biopsies of the prostate within a population-based screening program. Urol, 2002; 60(5): 826-30.

*9. Grant N, Stemmermann, Abraham M, Nomura Y, Chyou PH, and Yatani R. Prospective Comparison of Prostate Cancer at Autopsy and as a Clinical Event: The Hawaii Japanese experience. Cancer Epidemiol Biomarkers Prev, 1992; 1: 189-95.

*10. Chen Z, Trotman LC, Schaffer D, Lin HK, Do$\tan$ ZA, Niki M. et al. Crucial role of p53-dependent cellular senescence in suppression of Pten-deficient tumorigenesis. Nature. 2005; 436(7051):725-30.

11. Moul JW, Bauer JJ, Scrivastava S, Colon E, Ho CK, Sesterhenn IA, et al. Perineal seeding of prostate cancer as the only evidence of clinical recurrence 14 years after needle biopsy and radical prostatectomy: molecular correlation. Urol, 1998; 51: 158,162 .

12. Koppie TM, Grady BP, Shinohara K. Rectal wall recurrence of prostatic adenocarcinoma. Journal of Urol, 2002; 168: 2120-25.

*13. Bastacky SS, Walsh PC, Epstein JI. Needle biopsy associated tumor tracking of adenocarcinoma of the prostate. Journal of Urol, 1991; 145: 1003-7.

14. Valle Gerhold J, Azúa Blanco V, Gómez Aracil V, Villarroya Rodríguez S y Romeo Casabona P. Valor pronóstico de la citología en el cáncer de próstata en relación con la supervivencia. Act Urolo Esp, 1996; 20(4): 330-335.

*15. Bill-Axelson A, Holmberg L, Frej F, Ruutu M, Garmo H, Busch C. et al. Radical prostatectomy versus watchful waiting in localized prostate cancer: the Scandinavian prostate cancer group-4 randomized trial. J Natl Cancer Inst 2008;100(16):1144-54.

16. Eastham JA, Scardino P.T.: Radical prostatectomy. En En Campbell's Urology, ed. W.B. Saunders Company, Philadelphia, 1998, $7^{\text {a }}$ ed. pag 2551- 2556.

17. Litwin MS, Hays RD, Fink A, Ganz PA, Leake B, Leach GE, et al. Quality of life outcomes in mens treated for localiced prostate cancer. JAMA, 1995; 273: 129-35.

18. Klotz L. Active surveillance for prostate cancer: For whom? J Clin Oncol. 2005 23: 8165-8169.

19. Kenneth L, Lipsitz R, Miller T, Janakiraman S. Benefits and Harms of Prostate-Specific AntigenScreening for Prostate Cancer: An Evidence Update for the U.S. Preventive Services Task Force http://www.ahrq.gov/clinic/uspstf/uspsprca. htm

20. Mandelblatt JS, Cronin KA, Bailey S. Effects of Mammography Screening Under Different Screening Schedules: Model Estimates of Potential Benefits and Harms. Ann Intern Med, 2009;151: 738-47.

21. Hartmann KE, Hall SA, Nanda K, Boggess JF, Zolnoun D. Screening for Cervical Cancer, Topic Page. January 2003. U.S. Preventive Services Task Force. Agency for Healthcare Research and Quality, Rockville, MD. http://www.ahrq.gov/clinic/uspstf/uspscerv.htm

*22. Nieder AM, Porter MP, and Soloway MS. Radiation Therapy for Prostate Cancer Increases Subsequent Risk of Bladder and Rectal Cancer: A Population Based Cohort Study. J Urol, 2008; 180, 2005-10.

23. Wilt TJ, Brawer MK, Barry MJ, Jones KM, Kwon $\mathrm{Y}$, et al. The Prostate cancer Intervention Versus Observation Trial:VA/NCI/AHRQ Cooperative Studies Program 407 (PIVOT): design and baseline results of a randomized controlled trial comparing radical prostatectomy to watchful waiting for men with clinically localized prostate cancer. Contemp Clin Trials, 2009; 30(1):81-7. 